Supporting Information

\title{
Highly Deformable Transparent Au Film Electrodes and Their Uses in Deformable Displays
}

Dong Wook Kim, Gilwoon Lee, Monalisa Pal,* and Unyong Jeong*

Department of Materials Science and Engineering, Pohang University of Science and Technology (POSTECH), 77 Cheongam-Ro, Nam-Gu, Pohang, Gyeongbuk 37673, Republic of Korea

*Corresponding author: monalisa@,postech.ac.kr, ujeong@postech.ac.kr 


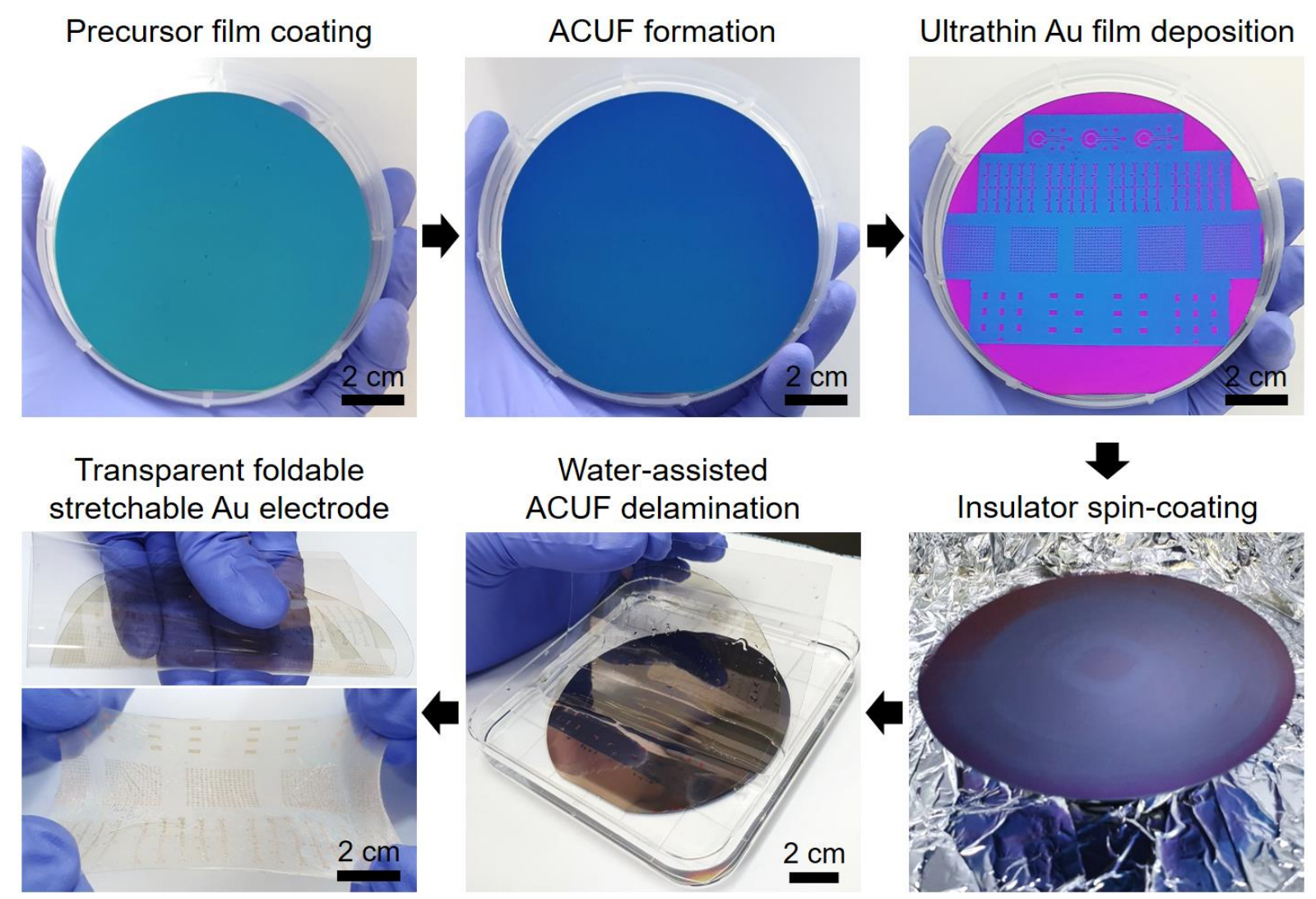

Figure S1. Photographs showing the fabrication process of the transparent foldable and stretchable ultrathin $\mathrm{Au}$ patterned electrode on a 4 inch $\mathrm{SiO}_{2} / \mathrm{Si}$ wafer using the ACUF. 

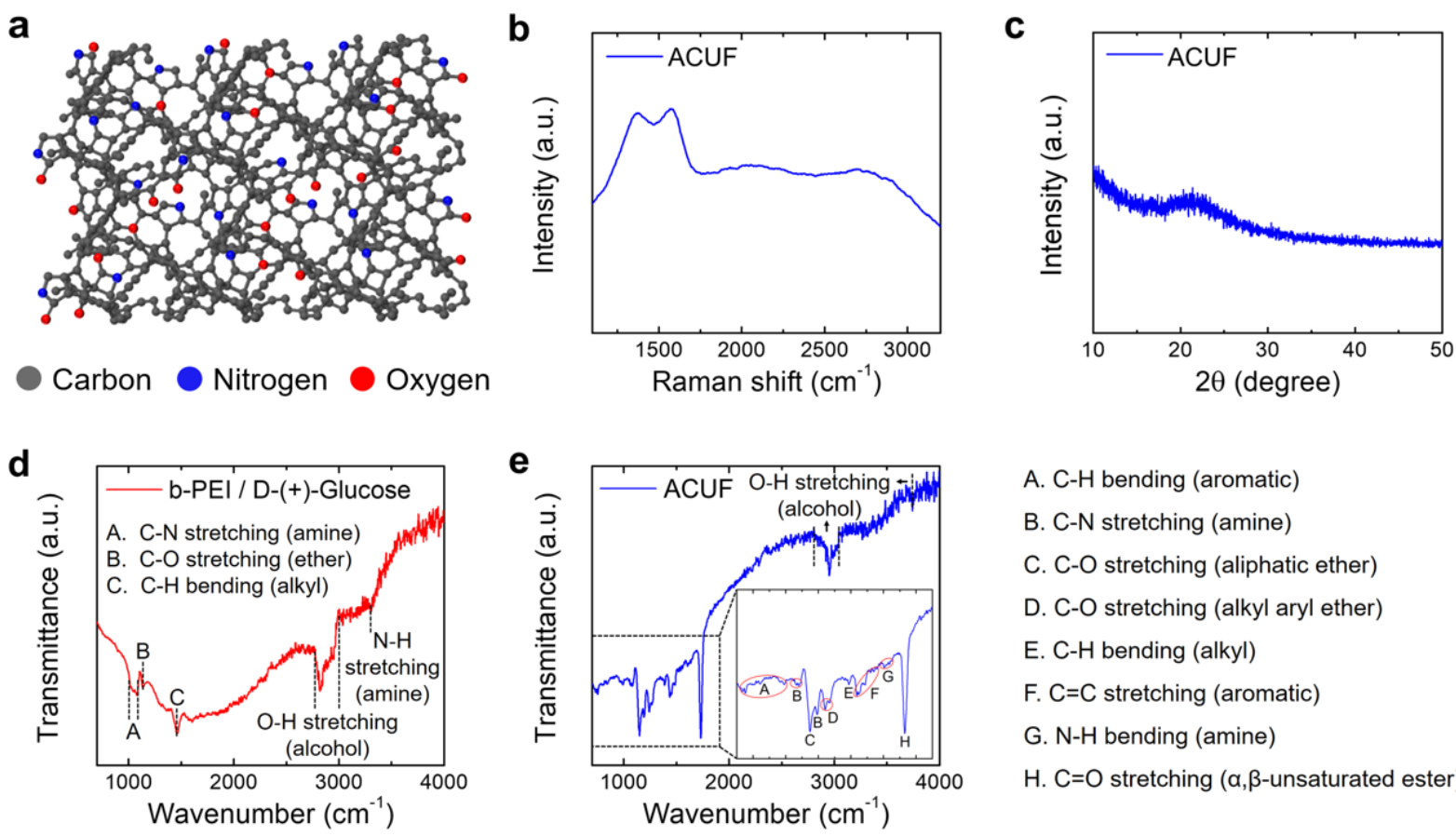

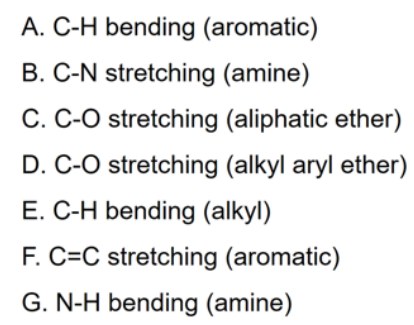
A. $\mathrm{C}-\mathrm{H}$ bending (aromatic)
B. C-N stretching (amine)
C. C-O stretching (aliphatic ether)
D. C-O stretching (alkyl aryl ether)
E. C-H bending (alkyl)
G. $\mathrm{N}-\mathrm{H}$ bending (amine)
H. C=O stretching ( $\alpha, \beta$-unsaturated ester)
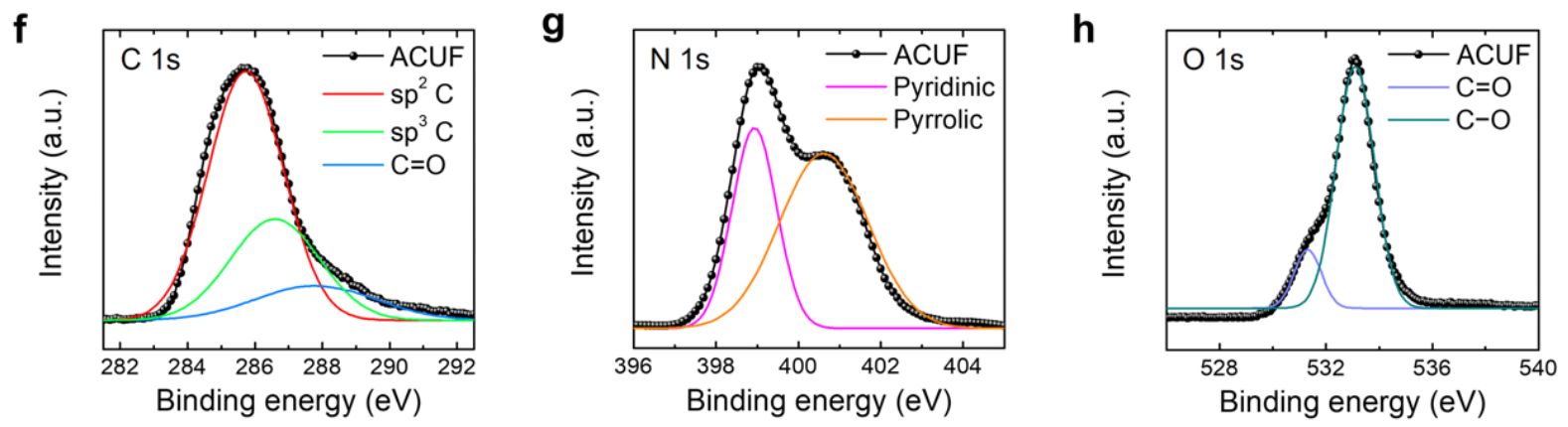

Figure S2. Characterization of the anisotropic conductive ultrathin film (ACUF). (a) Schematic illustration of the ACUF. (b-c) Raman (b) and X-ray diffraction (XRD) (c) spectra of the ACUF. (d-e) Fourier-transform infrared (FT-IR) spectra of the b-PEI/D-(+) glucose precursor film (d) and the ACUF (e). (f) C $1 \mathrm{~s}$ X-ray photoelectron spectroscopy (XPS) spectrum of the ACUF (black dots line) and its deconvolution with three types of C bonds: $\mathrm{sp}^{2}$ $\mathrm{C}$ (red), $\mathrm{sp}^{3} \mathrm{C}$ (green), and $\mathrm{C}=\mathrm{O}$ (blue). (g) $\mathrm{N}$ 1s XPS spectrum of the ACUF (black dots line) and its deconvolution with two types of $\mathrm{N}$ bonds: pyridinic (magenta) and pyrrolic (orange) bonds. (h) O 1s XPS spectrum of the ACUF (black dots line) and its deconvolution with two types of $\mathrm{O}$ bonds: $\mathrm{C}=\mathrm{O}$ (light purple) and $\mathrm{C}-\mathrm{O}$ (dark cyan) bonds. 


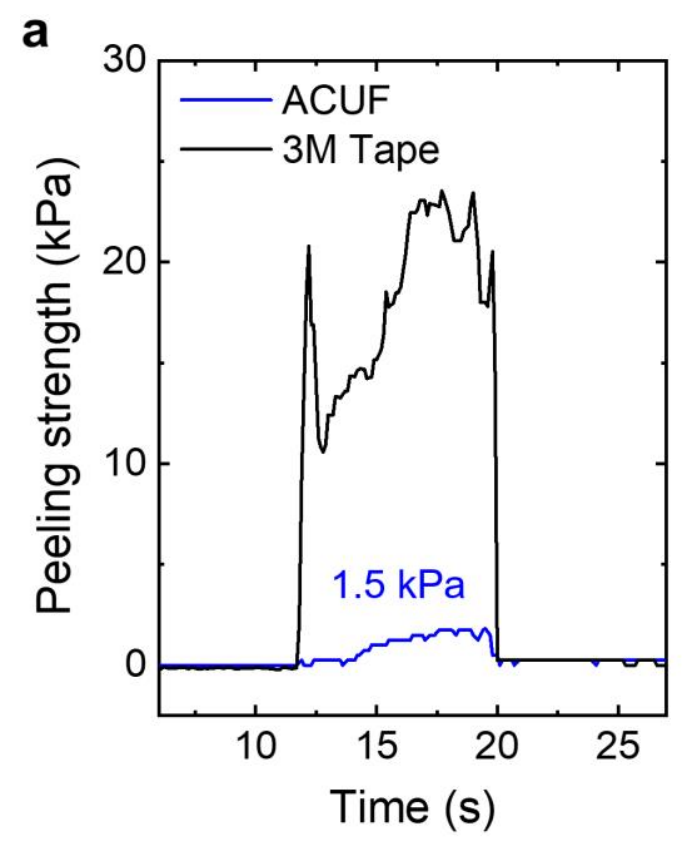

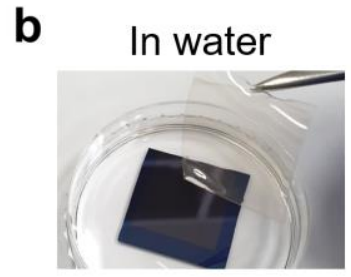

In dry air

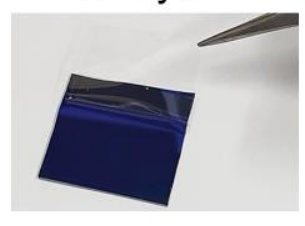

Ultrasonication

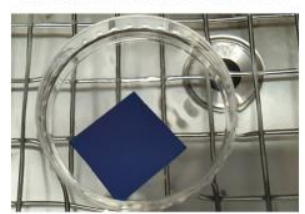

C

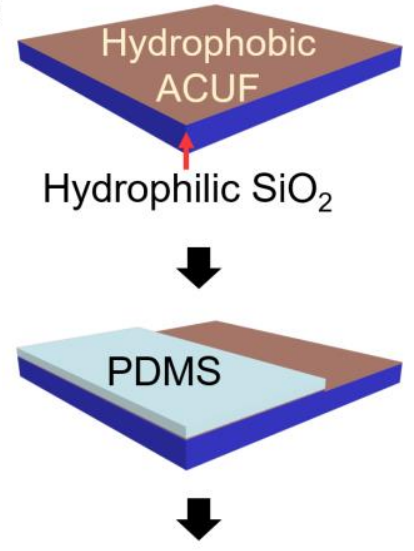

Delamination in water

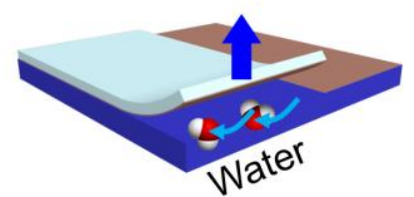

Figure S3. Water-assisted delamination of the ACUF. (a) Peeling strength required to delaminate the ACUF using a PDMS film and to peel off the commercial $3 \mathrm{M}$ tape from a $\mathrm{SiO}_{2} / \mathrm{Si}$ wafer. (b) Photographs of the ACUF which was delaminated using a PDMS film in water (top) but kept intact under dry air (middle). The bottom image is the photograph of the ACUF under ultrasonication in water. (c) Schemes for the principle of ACUF delamination in water. 

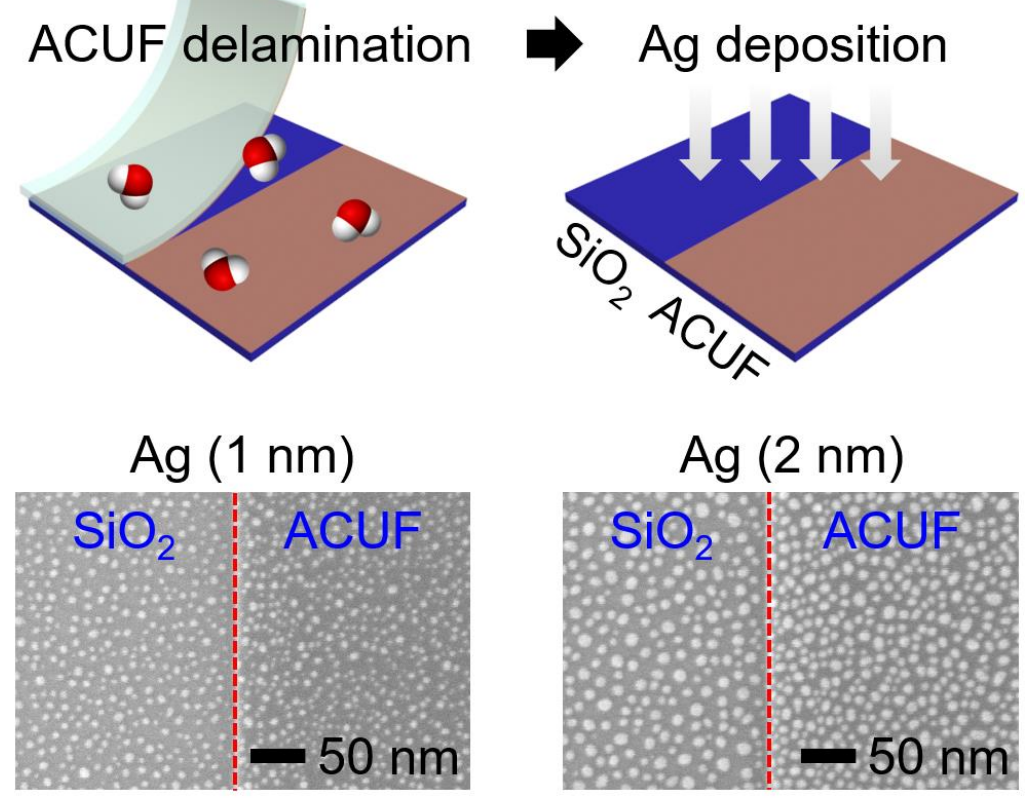

$$
\operatorname{Ag}(3 \mathrm{~nm})
$$
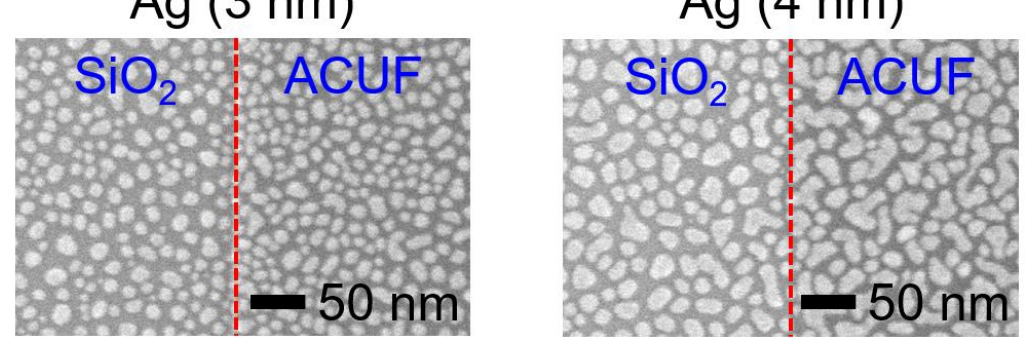

Figure S4. Effect of the ACUF on the Ag deposition. SEM images of the boundary between $\mathrm{SiO}_{2}$ and ACUF with different thickness of Ag. The boundary between the $\mathrm{SiO}_{2}$ and ACUF was made by delaminating half of the ACUF using PDMS films. Compared to the $\mathrm{SiO}_{2}$ side, denser and smaller Ag islands were formed on the ACUF side. 


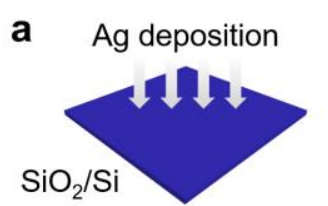

b Ag deposition

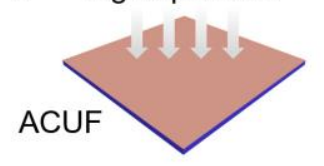

C Au deposition

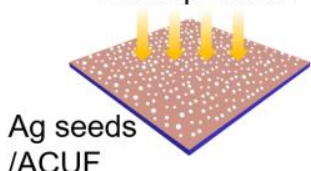

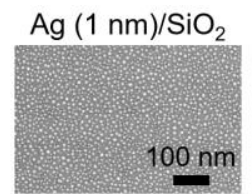

$\mathrm{Ag}(5 \mathrm{~nm}) / \mathrm{SiO}_{2}$
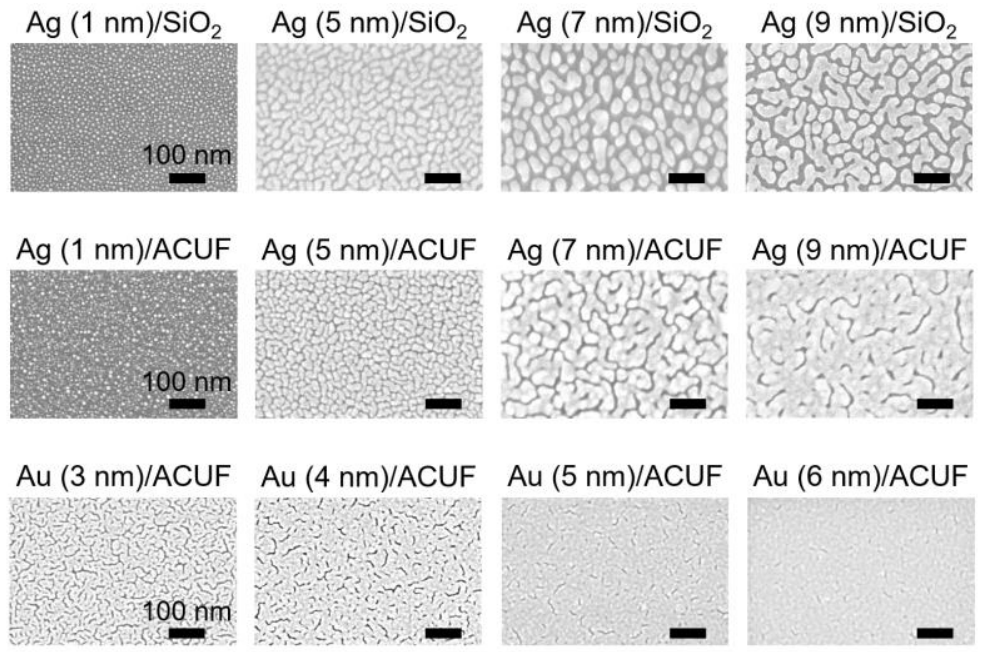

$\mathrm{Ag}(10 \mathrm{~nm}) / \mathrm{SiO}_{2}$

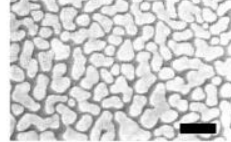

Ag $(7 \mathrm{~nm}) / \mathrm{ACUF} \quad \mathrm{Ag}(9 \mathrm{~nm}) / \mathrm{ACUF} \quad \mathrm{Ag}(10 \mathrm{~nm}) / \mathrm{ACUF}$
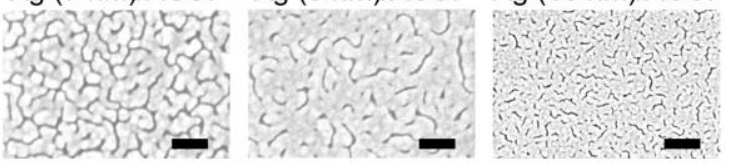

Au $(5 \mathrm{~nm}) / \mathrm{ACUF} \quad \mathrm{Au}(6 \mathrm{~nm}) / \mathrm{ACUF} \quad \mathrm{Au}(7 \mathrm{~nm}) / \mathrm{ACUF}$

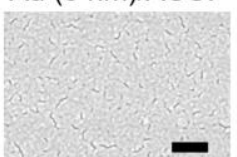

Figure S5. SEM images of the metal deposited film surfaces. (a) Surface SEM images of the Ag-deposited $\mathrm{SiO}_{2} / \mathrm{Si}$ wafer $\left(\mathrm{Ag} / \mathrm{SiO}_{2}\right)$ with different thickness of Ag. (b) Surface SEM images of the Ag-deposited ACUF (Ag/ACUF) with different thickness of Ag. (c) Surface SEM images of the Au-deposited Ag seeds (1 nm)/ACUF (Au/ACUF) with different thickness of Au. 


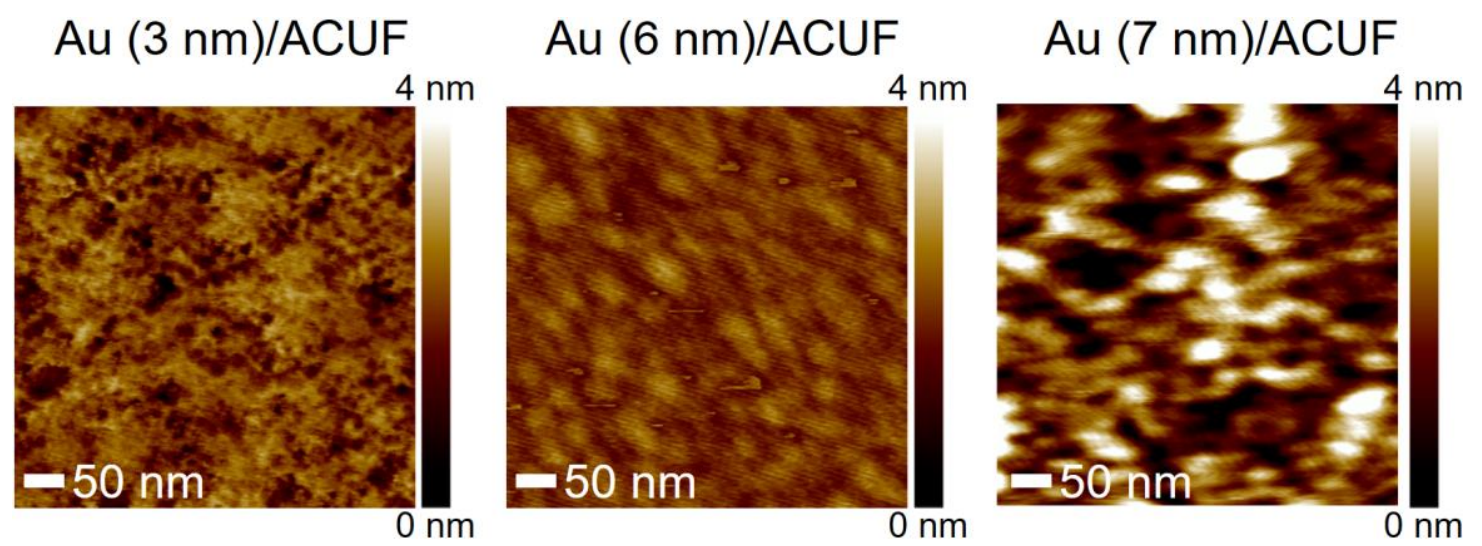

Figure S6. AFM images showing the surface morphology of the Au/ACUF at $t_{\mathrm{Au}}=3,6$, and $7 \mathrm{~nm}$. 
a

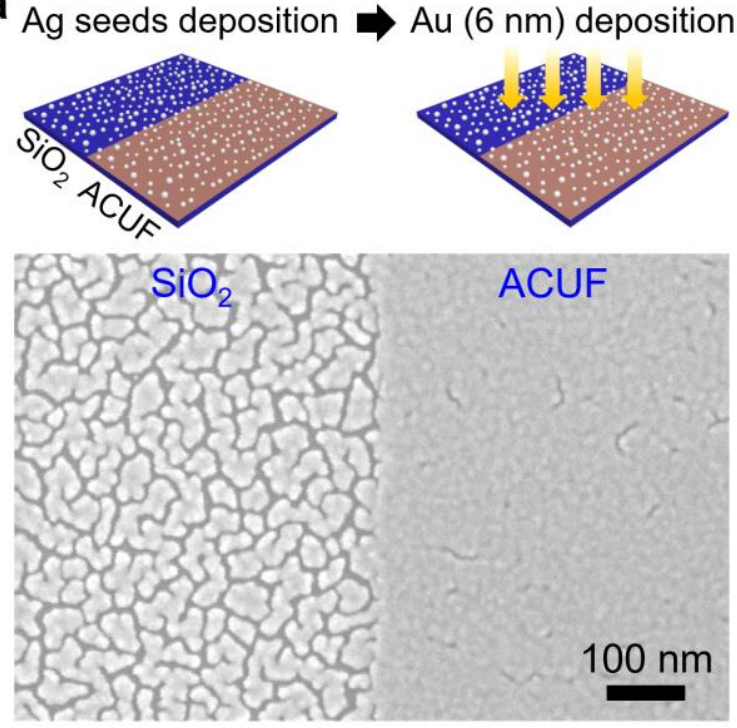

b

$\mathrm{Au}(10 \mathrm{~nm})$ deposition
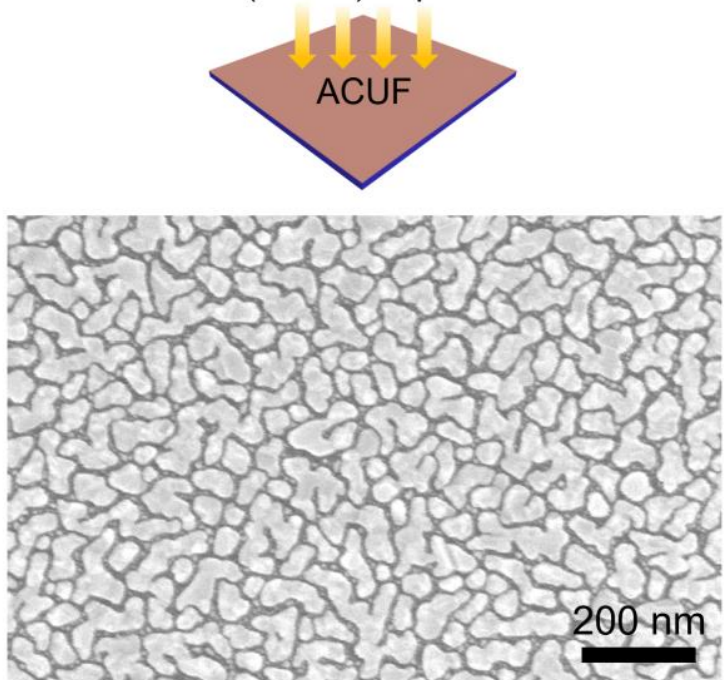

Figure S7. Effect of the Ag seeds and the ACUF on Au deposition. (a) SEM image of the $\mathrm{SiO}_{2} / \mathrm{ACUF}$ boundary with $\mathrm{Ag}$ seeds $(1 \mathrm{~nm})$ and $6 \mathrm{~nm}$ Au layer. The $\mathrm{Ag}$ seeds allow the formation of continuous ultrathin Au film only on the ACUF surface. (b) SEM image of the 10 nm Au-deposited ACUF. Without the Ag seeds, a conductive Au film was not formed on the ACUF. These results indicate that the ACUF and the Ag seeds collaboratively helped the formation of the continuous ultrathin Au film. 


Water Ethylene glycol \begin{tabular}{lr}
\multicolumn{2}{c}{ Contact angle (degree) on ACUF } \\
\cline { 3 - 4 }
\end{tabular}

- Geometric mean equation

$\gamma_{1}(1+\cos \theta)=2\left(\gamma_{\mathrm{s}}^{\mathrm{d}} \gamma_{1}^{\mathrm{d}}\right)^{1 / 2}+2\left(\gamma_{\mathrm{s}}^{\mathrm{p}} \gamma_{\mathrm{l}}^{\mathrm{p}}\right)^{1 / 2}$

Deionized water : $\gamma_{\text {DIW }}{ }^{\mathrm{d}}: 21.8 \mathrm{~mJ} \mathrm{~m}^{-2} \quad \gamma_{\text {DIW }}{ }^{\mathrm{p}}: 51.0 \mathrm{~mJ} \mathrm{~m}^{-2}$

Ethylene glycol : $\gamma_{\mathrm{EG}}{ }^{\mathrm{d}}: 29.3 \mathrm{~mJ} \mathrm{~m}^{-2} \quad \gamma_{\mathrm{EG}}^{\mathrm{p}}: 19.0 \mathrm{~mJ} \mathrm{~m}^{-2}$

\begin{tabular}{ccc}
\multicolumn{3}{c}{ ACUF surface energy $\left(\mathrm{mJ} \mathrm{m}^{-2}\right)$} \\
\hline$\gamma^{\mathrm{d}}$ & $\gamma^{\mathrm{p}}$ & $\gamma^{\text {total }}$ \\
\hline 23.3 & 19.1 & 42.4
\end{tabular}

\begin{tabular}{ccc}
\multicolumn{3}{c}{ Surface energy $\left(\mathrm{mJ} \mathrm{m}^{-2}\right)$} \\
\hline ACUF & Silver & Gold \\
\hline 42.4 & 1250 & 1500
\end{tabular}

Figure S8. ACUF surface energy calculation and comparison with metals. The ACUF surface energy was calculated from contact angle measurements performed with distilled water and ethylene glycol droplets by applying the geometric mean equation, where $\gamma_{1}$ and $\gamma_{\mathrm{s}}$ are the surface energies of the liquid and the substrate, respectively. The $\gamma^{\mathrm{d}}$ and $\gamma^{\mathrm{p}}$ refer to the dispersion and polar surface energy, respectively. The total surface energy $\left(\gamma^{\text {total }}\right)$ is the sum of $\gamma^{\mathrm{d}}$ and $\gamma^{\mathrm{p}}$. 


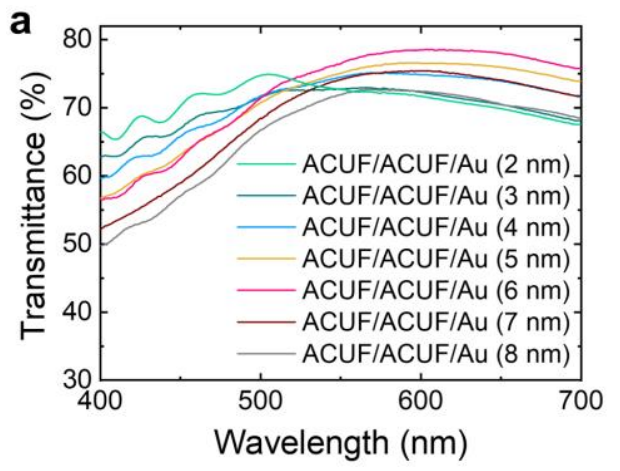

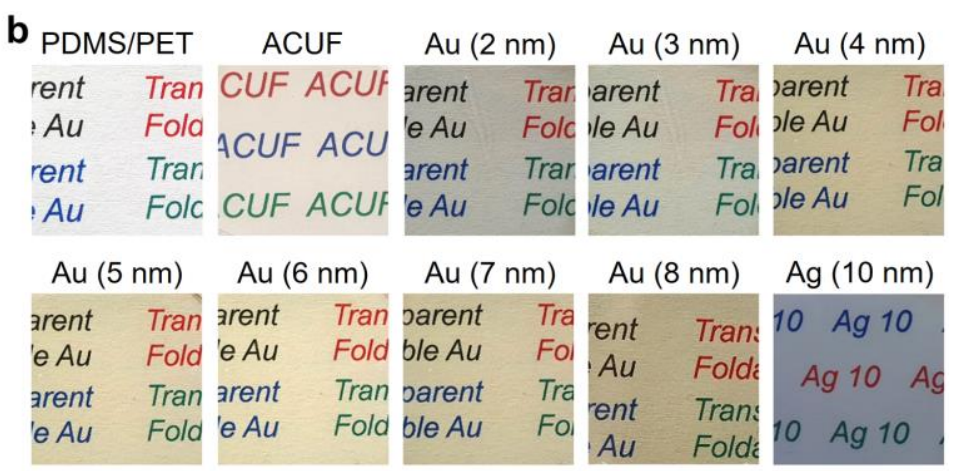

Figure S9. Transmittance spectra and photographs of the ACUF/ACUF/Au electrodes. (a) Transmittance spectra of the ACUF/ACUF/Au transparent electrodes with Au films of different thickness. (b) Photographs of the PDMS/PET substrate, ACUF, and ACUF/ACUF/Au transparent electrodes with Au films of different thickness. Photograph of the ACUF/ACUF/Ag $(10 \mathrm{~nm})$ electrode was also given for comparison. The colored letters was placed below the sample for transparency comparison. The size of the photograph is $2.5 \mathrm{~cm} \times 2.5 \mathrm{~cm}$. 

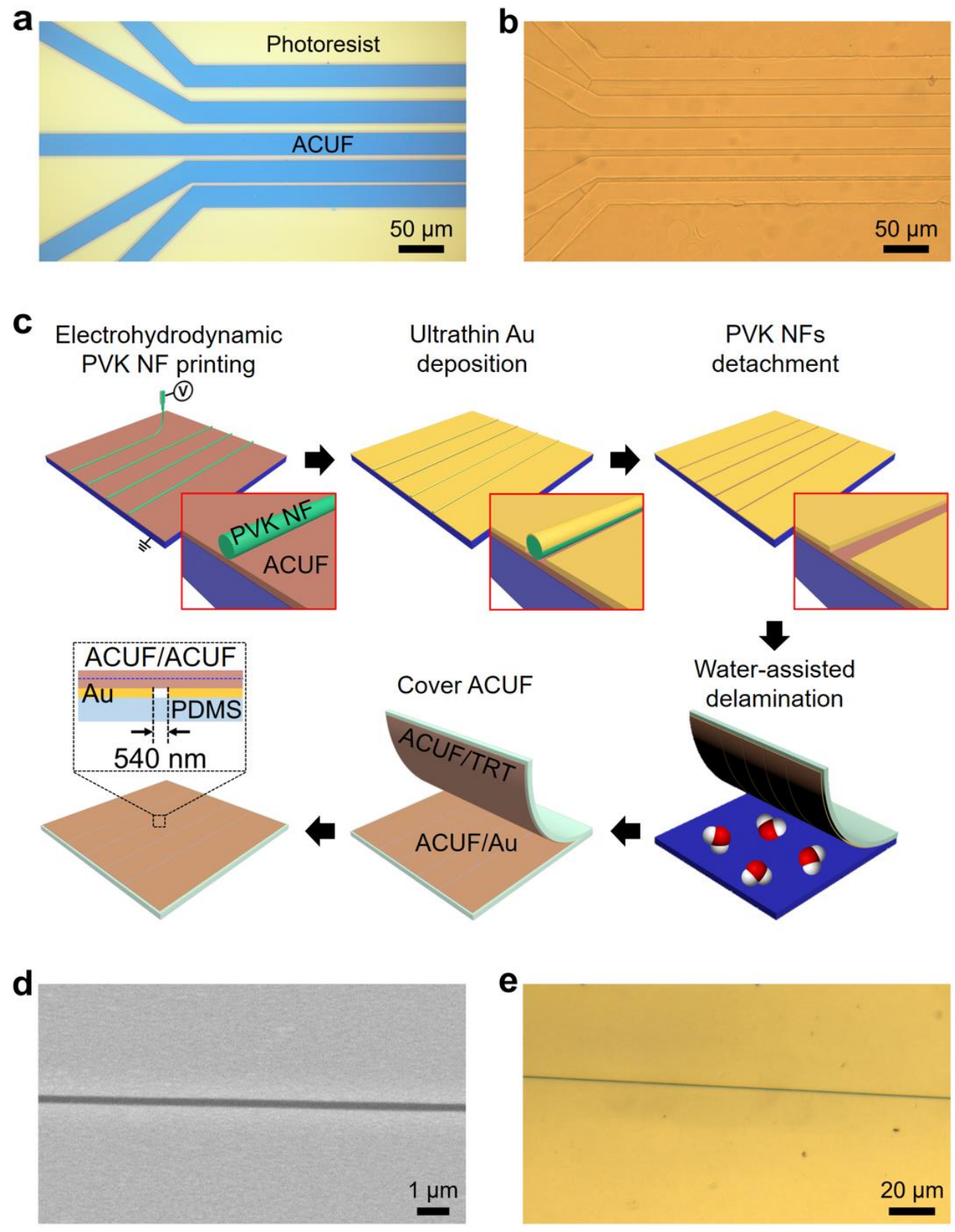

Figure S10. ACUF/ACUF/Au electrode with different gaps in Au layer. (a, b) OM image of the photoresist pattern formed on the ACUF (a) and the ACUF/ACUF/Au electrode with different gaps in the Au layer (b). (c) Schemes for the nanofiber (NF) lithography process. The poly(9-vinylcarbazole) (PVK) NFs were first printed on the ACUF by electrohydrodynamic NF printing. Then, $6 \mathrm{~nm}$ Au was deposited on the sample surface and the Au coated-PVK NFs were detached through taping detachment. The ACUF/Au layer with nanogaps in the Au layer was transferred to the PDMS/PET substrate. Finally, another ACUF was transferred to the sample. (d) SEM image of the Au/ACUF electrode with nanogap Au patterns. (e) OM image of the ACUF/ACUF/Au electrode with nanogap Au patterns. 

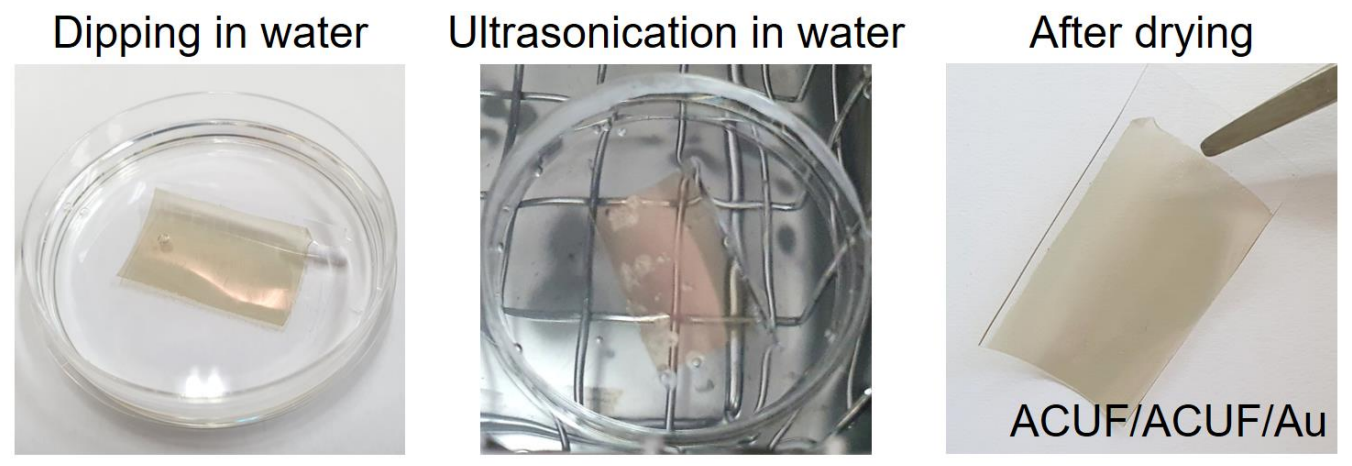

Figure S11. Stability of the ACUF layer in the ACUF/ACUF/Au electrode. Although the adhesion between the ACUF layers did not contain strong bonds, the ACUF cover layer was not peeled off by dipping it in water or applying ultrasonication in water. 

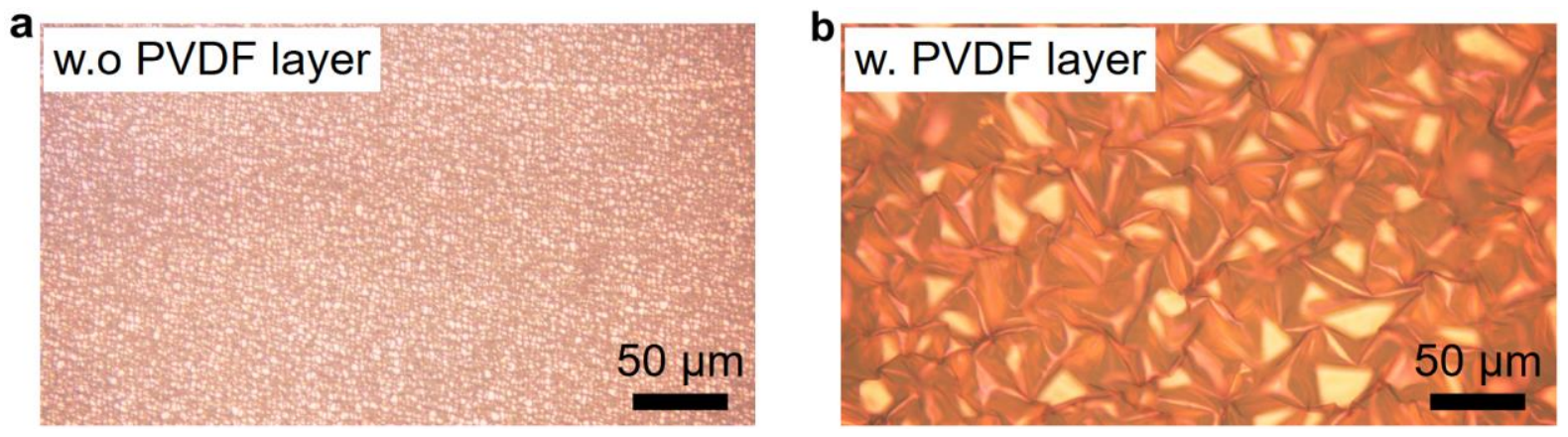

Figure S12. (a, b) OM images of the ACUF/Au film after being crumpled onto the PDMS substrates with (a) and without (b) the PVDF layer. The tough PVDF layer prevented the rupture of the ACUF/Au film while handling the electrode or releasing the pre-strain in the PDMS substrate. Without the PVDF layer, the ACUF/Au film ruptured after being transferred onto the PDMS substrate. 


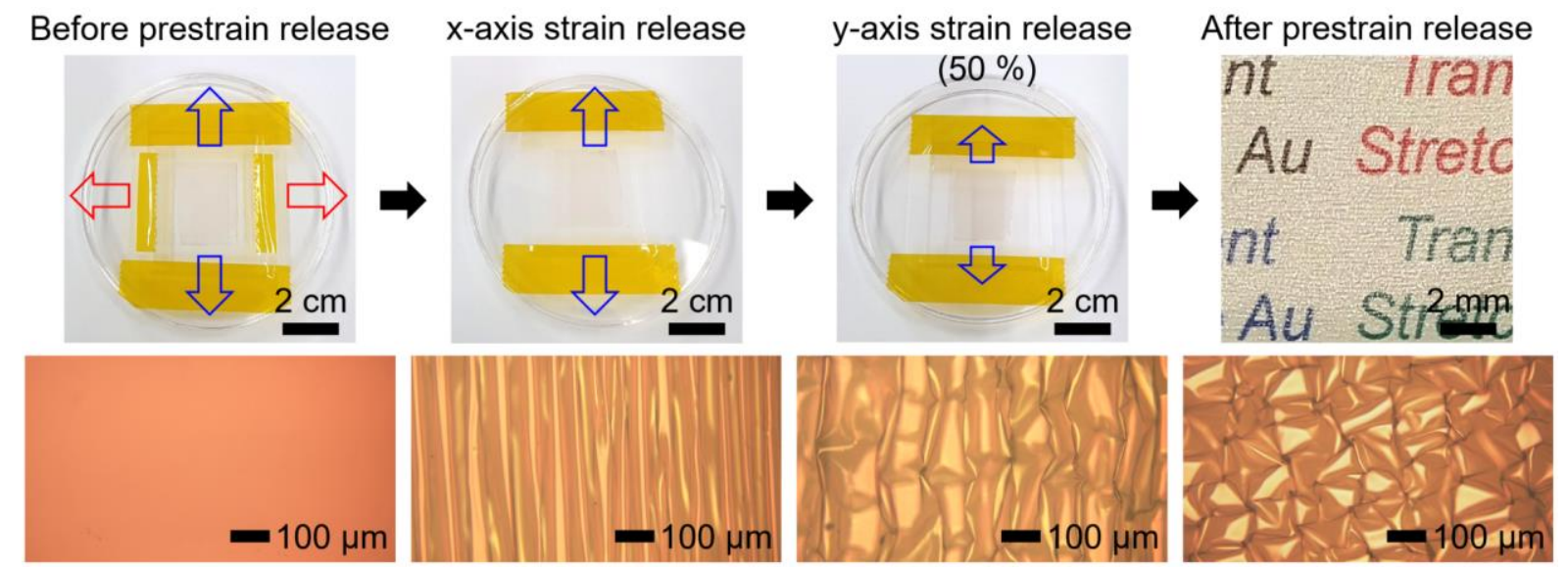

Figure S13. Fabrication process of the ACUF/ACUF/Au transparent stretchable electrode. Photographs and $\mathrm{OM}$ images showing the fabrication process of the transparent stretchable ACUF/ACUF/Au electrode. By releasing the pre-strain in the PDMS substrate gradually, the flat ACUF/ACUF/Au electrode on the PDMS substrate became crumpled. 

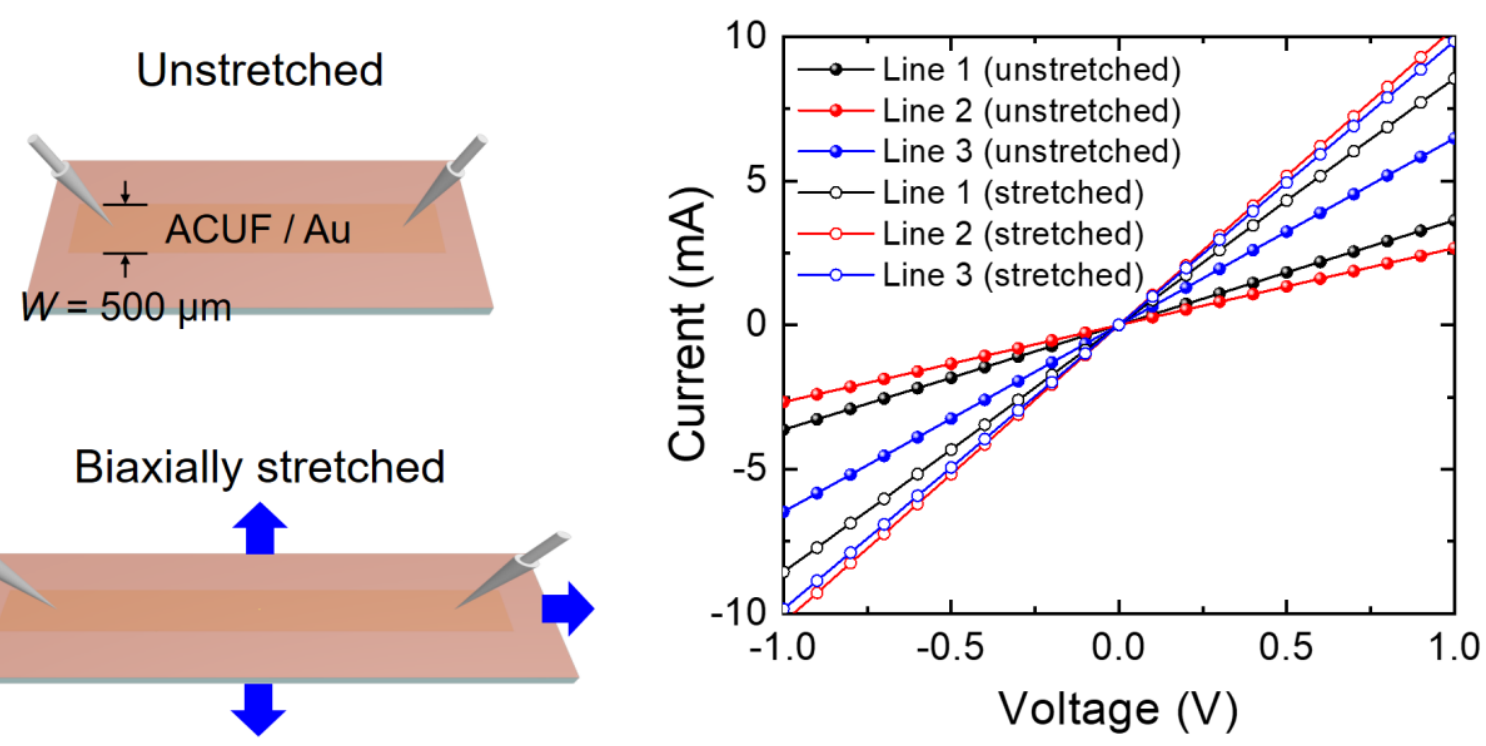

Figure S14. Current-voltage curves of the line-patterned stretchable ACUF/Au electrodes (500 $\boldsymbol{\mu m}$ width) in unstretched and biaxially stretched (50\% strain) states. Current was measured from three different ACUF/Au line electrodes. Without the additional ACUF cover, the current could not be reproduced at each electrode and was changed by stretching. 


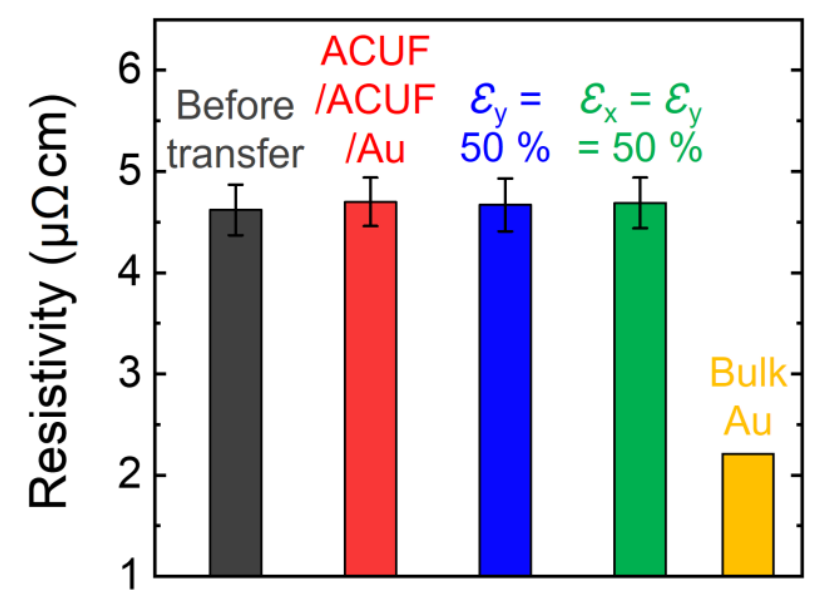

Figure S15. Comparison of the resistivity of the evaporated Au pattern (50 $\mu \mathrm{m}$ width) before transfer (black), the same pattern in the ACUF/ACUF/Au without stretching (red), uniaxial stretching (blue), and biaxial stretching (green). The resistivity of the bulk Au (yellow) is shown for comparison. 

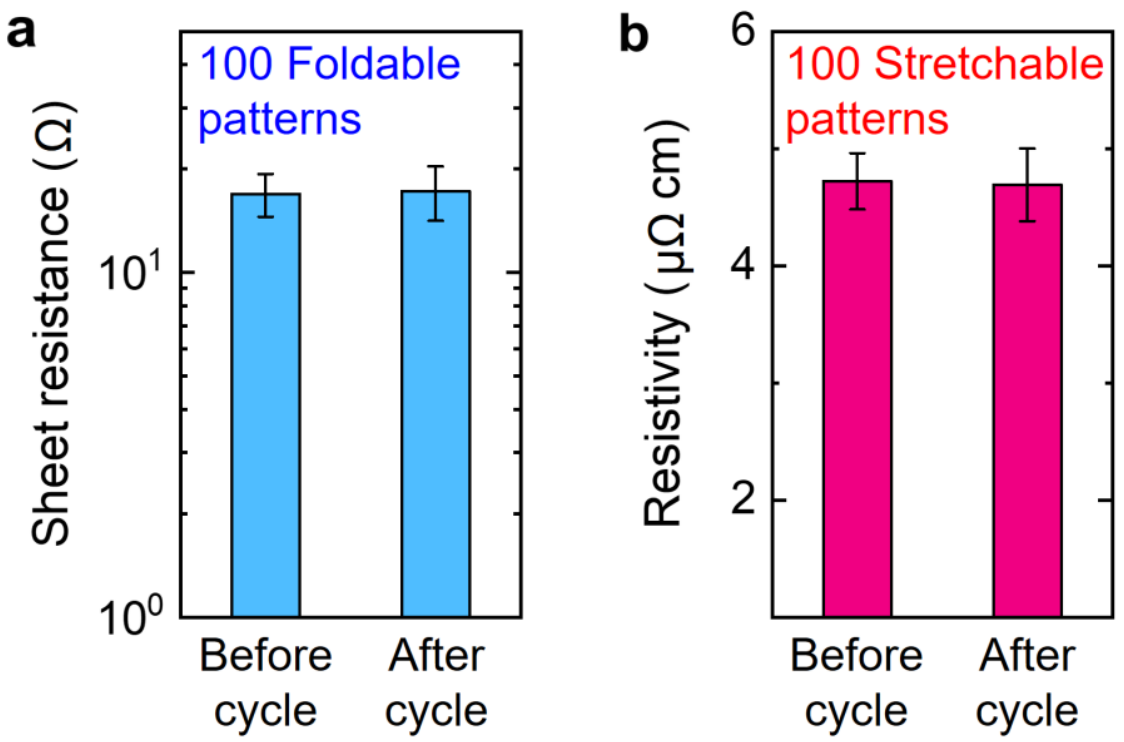

Figure S16. (a) $R_{\mathrm{s}}$ change of the 100 foldable ACUF/ACUF/Au patterns which was randomly measured from the folded region of the 4 inch foldable electrode. (b) Resistivity change of the 100 stretchable ACUF/ACUF/Au lines were randomly measured from a large-area stretchable electrode $(10 \mathrm{~cm} \times 10 \mathrm{~cm}$ size). The number of deformation (folding and stretching) cycles was 3000 . 

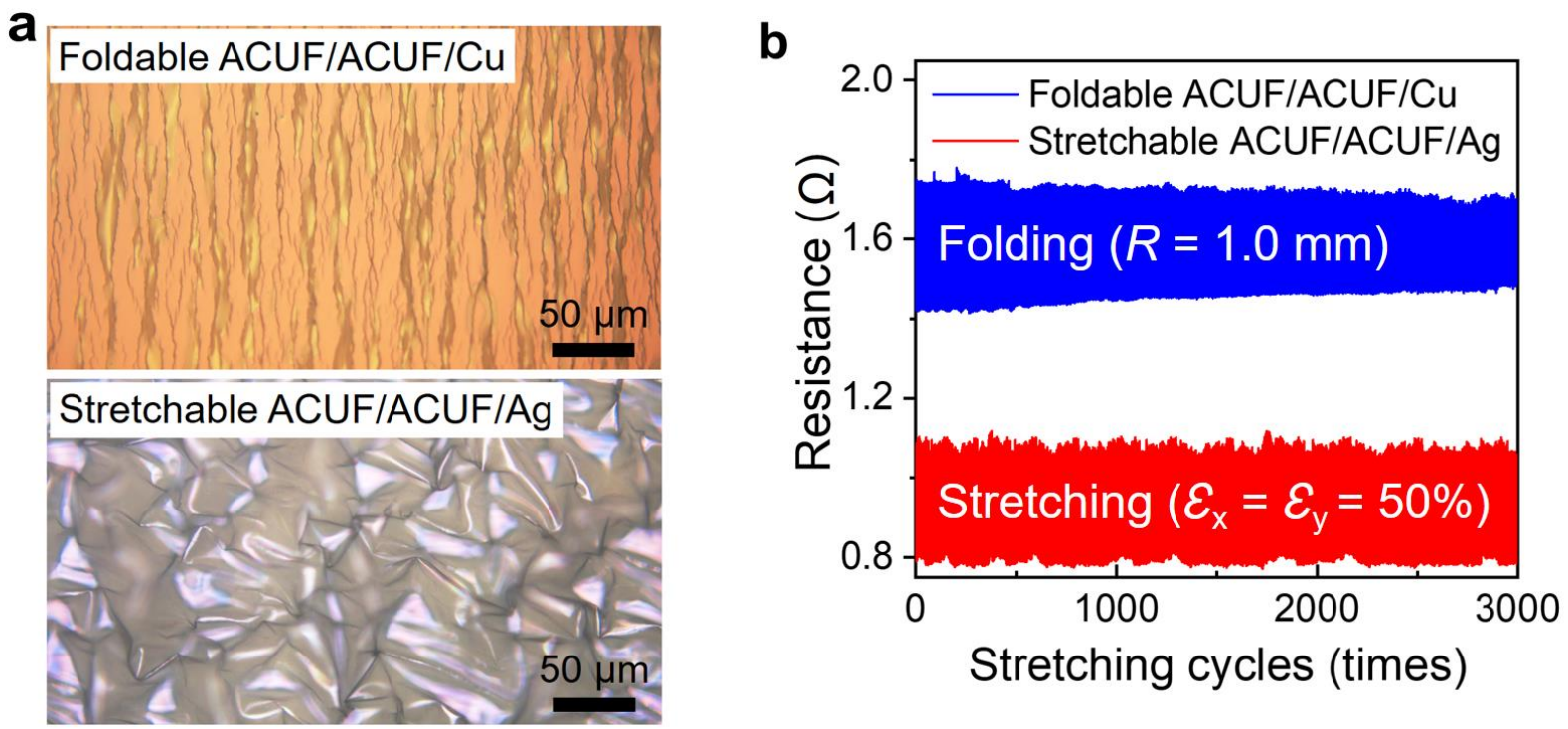

Figure S17. (a) $\mathrm{OM}$ images of the foldable $\mathrm{ACUF} / \mathrm{ACUF} / \mathrm{Cu}(20 \mathrm{~nm})$ electrode and the stretchable ACUF/ACUF/Ag $(20 \mathrm{~nm})$ electrode after folding/stretching cycles. (b) The electrical stability of the electrodes while applying 3000 cycles of folding $(R=1.0 \mathrm{~mm})$ and biaxial stretching $\left(\varepsilon_{\mathrm{x}}=\varepsilon_{\mathrm{y}}=50 \%\right)$. 
a
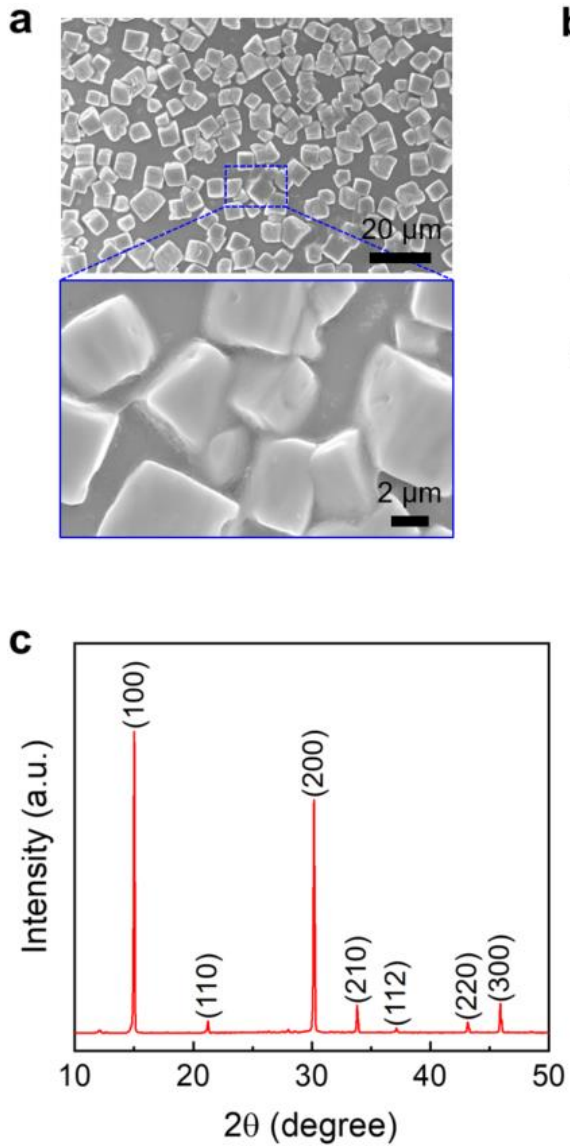
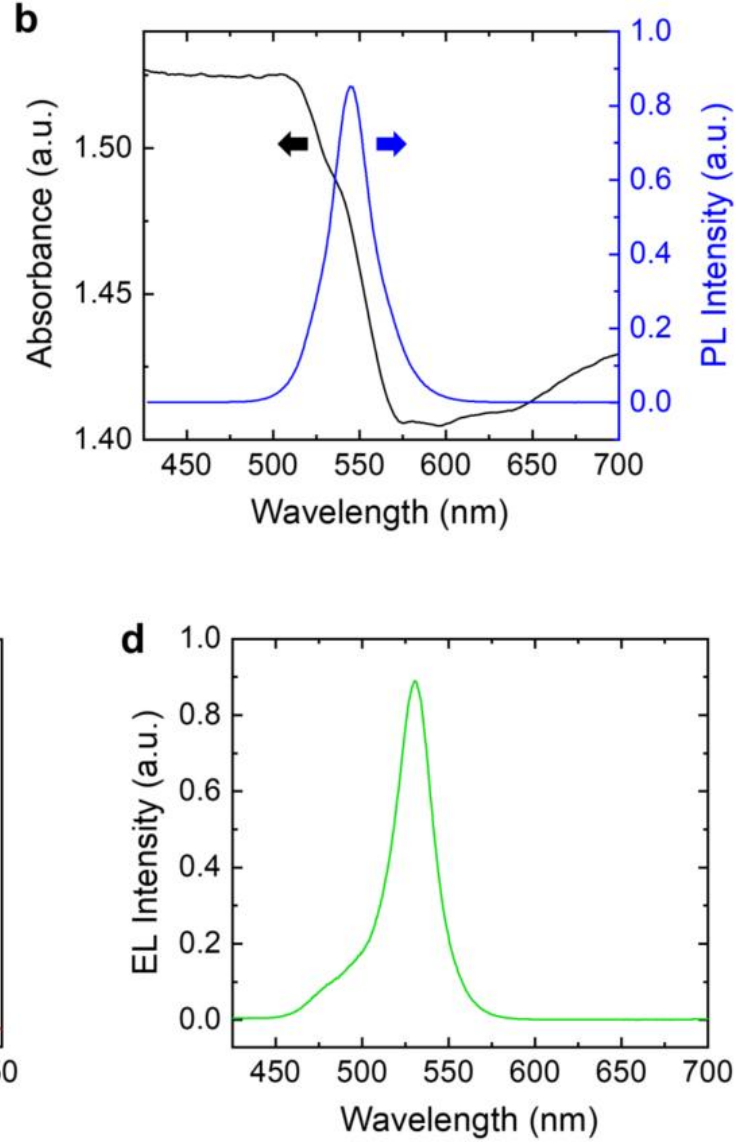

Figure S18. Characterization of the PeLED. (a) Surface SEM image of the MAPbBr $3: \mathrm{PEO}$ composite film. The $\mathrm{MAPbBr}_{3}$ crystals were embedded into the PEO matrix. (b) Photoluminescence (PL) and absorbance spectra of the MAPbBr3:PEO composite film. (c) $\mathrm{XRD}$ pattern of the MAPbBr3:PEO composite film. (d) Electroluminescence (EL) spectrum of the PeLED at a $5 \mathrm{~V}$ bias. 

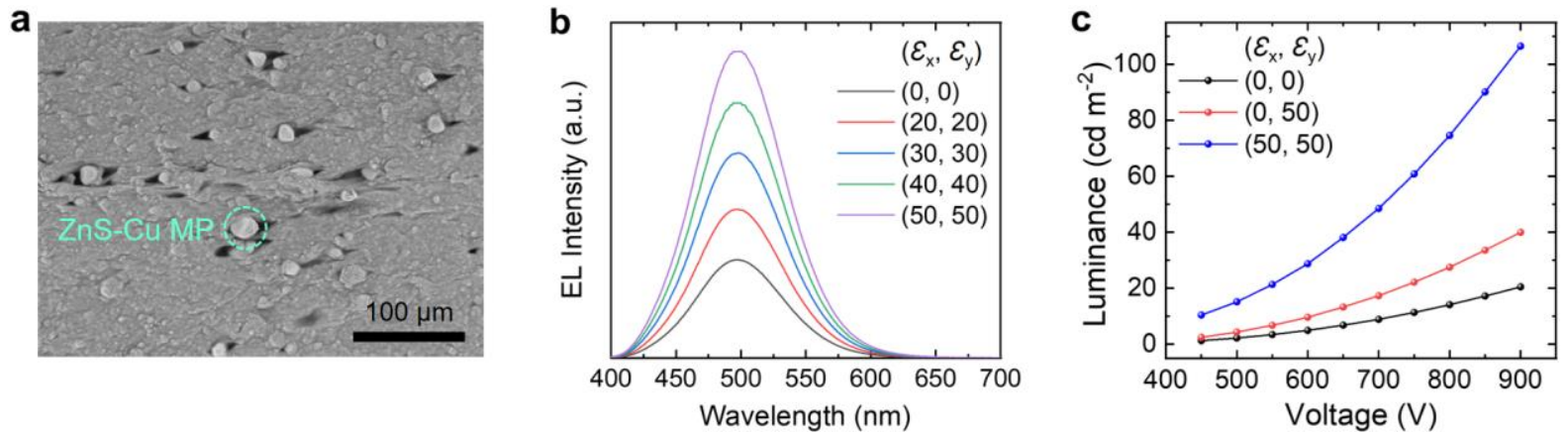

Figure S19. Characterization of the ACEL device. (a) Cross-sectional SEM image of the $\mathrm{ZnS}-\mathrm{Cu}$ :PDMS composite film. (b) EL spectra of the stretchable ACEL device at different biaxial strain. (c) Luminance-pulse voltage characteristic of the ACEL device at different strain. 


\section{Supporting Movies}

Movie S1. Peeling test of the ACUF and ultrasonication of the ACUF in water.

Movie S2. Lit foldable perovskite LED and folding at $R=1.0 \mathrm{~mm}$ and $R=0.5 \mathrm{~mm}$.

Movie S3. Stretching and twisting of the stretchable ACEL device and its pixel operation. 